\title{
Melioidosis in northern Sri Lanka: filling thegap in the map
}

\author{
FNMubarak $^{1}$, SThavapalan ${ }^{1}$, EMCorea ${ }^{2}$, AD De Silva ${ }^{3}$, BJRCooray ${ }^{4}$, SGhetheeswaran ${ }^{5}$,

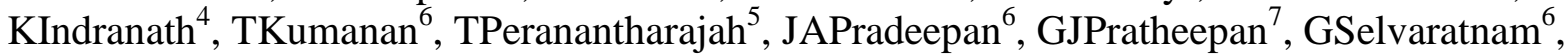 \\ SSivansuthan $^{5}$, NSuganthan ${ }^{6}$, SUthayakumaran ${ }^{5}$
}

\section{Introduction}

National surveillance has established melioidosis as endemic throughout Sri Lanka but data is lacking from the Northern Province. Only one culture-positive case had been reported up to 2013, probably due to lack of clinical microbiology services and misidentification of Burkholderiapseudomallei as Pseudomonas species due to lack of technical familiarity. This study describes melioidosis in northern Sri Lanka between April 2014 and April 2017.

\section{Methods}

Clinical microbiology services at Teaching Hospital, Jaffna were upgraded in 2014. B. pseudomallei culture work-up procedure was introduced with reference laboratory support. Technicians were trained using control cultures. Physician awareness was increased. Sample collection for culture and antibody detection from suspected patients was encouraged. Probable isolates were confirmed by real time PCR assays for LpxO and YLF/BTFC targets and serum was sent for antibody testing by the indirect haemagglutination assay.

\section{Results}

Six culture-positive cases were detected in 2016, three males and three females, all above 40 years of age. Clinical presentations included sepsis, pneumonia, septic arthritis and abscess. Four patients died (case fatality rate 67\%), one recovered and one was lost to follow-up. The causative organism was isolated from two knee joint $(\mathrm{KJ})$ aspirates and five blood cultures (both blood and KJ aspirate culture were positive in one patient). Five patients had significant risk factors for exposure such as rice farming $(n=2)$, flooding $(n=2)$ and construction work $(n=1)$. Three were diabetic and other comorbid conditions (alcoholism, malignancy) were present. Four isolates belonged to the Southeast Asian biogeographic YLF variant and two belonged to the Australian BTFC variant. Antibody titres tested in three of the culture-positive patients were $\geq 2560$.

Serum antibody titres were tested in 99 patients and 19 (19.2\%) had levels $\geq 1: 40$. Three (who remained culture-negative but had antibody titres of 320,>10240 and >10240 respectively) were treated for probable melioidosis and all recovered.

\footnotetext{
${ }^{1}$ Department of Microbiology and Infection Prevention \& Control, Teaching Hospital, Jaffna, Sri Lanka.

${ }^{2}$ Department of Microbiology, Faculty of Medicine, University of Colombo, Sri Lanka.

${ }^{3}$ Genetech Research Institute, Colombo Sri Lanka.

${ }^{4}$ Tellippalai Trail Cancer Hospital, Tellippalai, Sri Lanka.

${ }^{5}$ Medical Unit, Teaching Hospital, Jaffna, Sri Lanka.

${ }^{6}$ Department of Medicine, Faculty of Medicine, University of Jaffna, Sri Lanka.

${ }^{7}$ Medical Unit, District General Hospital, Killinochchi, Sri Lanka
}

Address for correspondence: Dr Nasmiya Mubarak, Department of Microbiology and Infection Prevention and Control, Teaching Hospital, Jaffna, Sri Lanka. +94 777518338 Email: nsmya@yahoo.com iD https://orcid.org/00000002-0103-1189 


\section{Discussion and Conclusions}

These findings address the dearth of information on melioidosis from northern Sri Lanka. Many cases probably remain undetected. Clinical and laboratory diagnosis should be continuously reinforced for improving case detection as early diagnosis has led to reduced mortality elsewhere in the country. 